\title{
Clinical Evaluation of Zero-Echo-Time MR Imaging for the Segmentation of the Skull
}

\author{
Gaspar Delso*1, Florian Wiesinger*2, Laura I. Sacolick ${ }^{2}$, Sandeep S. Kaushik ${ }^{3}$, Dattesh D. Shanbhag ${ }^{3}$, Martin Hüllner ${ }^{\dagger 4}$, \\ and Patrick Veit-Haibach ${ }^{\dagger}$ \\ ${ }^{1}$ MR Applications and Workflow, GE Healthcare, Waukesha, Wisconsin; ${ }^{2}$ GE Global Research, Munich, Germany; ${ }^{3}$ GE Global \\ Research, Bangalore, India; and ${ }^{4}$ Department of Medical Imaging, University Hospital, Zurich, Switzerland
}

MR-based attenuation correction is instrumental for integrated PET/ MR imaging. It is generally achieved by segmenting MR images into a set of tissue classes with known attenuation properties (e.g., air, lung, bone, fat, soft tissue). Bone identification with MR imaging is, however, quite challenging, because of the low proton density and fast decay time of bone tissue. The clinical evaluation of a novel, recently published method for zero-echo-time (ZTE)-based MR bone depiction and segmentation in the head is presented here. Methods: A new paradigm for MR imaging bone segmentation, based on proton density-weighted ZTE imaging, was disclosed earlier in 2014. In this study, we reviewed the bone maps obtained with this method on 15 clinical datasets acquired with a PET/CT/MR trimodality setup. The CT scans acquired for PET attenuation-correction purposes were used as reference for the evaluation. Quantitative measurements based on the Jaccard distance between ZTE and CT bone masks and qualitative scoring of anatomic accuracy by an experienced radiologist and nuclear medicine physician were performed. Results: The average Jaccard distance between ZTE and CT bone masks evaluated over the entire head was $52 \% \pm 6 \%$ (range, $38 \%-63 \%$ ). When only the cranium was considered, the distance was $39 \% \pm 4 \%$ (range, $32 \%-$ $49 \%)$. These results surpass previously reported attempts with dualecho ultrashort echo time, for which the Jaccard distance was in the $47 \%-79 \%$ range (parietal and nasal regions, respectively). Anatomically, the calvaria is consistently well segmented, with frequent but isolated voxel misclassifications. Air cavity walls and bone/fluid interfaces with high anatomic detail, such as the inner ear, remain a challenge. Conclusion: This is the first, to our knowledge, clinical evaluation of skull bone identification based on a ZTE sequence. The results suggest that proton density-weighted ZTE imaging is an efficient means of obtaining high-resolution maps of bone tissue with sufficient anatomic accuracy for, for example, PET attenuation correction.

Key Words: ZTE; bone; PET/MR; attenuation; skull

J Nucl Med 2015; 56:417-422

DOI: 10.2967/jnumed.114.149997

$\mathbf{H}$ ybrid PET/MR imaging is an emerging modality with potential applications in oncology, cardiology, and neurology. Since

Received Oct. 14, 2014; revision accepted Dec. 15, 2014.

For correspondence or reprints contact: Gaspar Delso, PET/CT-MR Center, University Hospital of Zurich, Wagistrasse 14, 8952 Schlieren, Switzerland.

E-mail: gaspar.delso@usz.ch

${ }^{*}$ Contributed equally to this work.

tContributed equally to this work.

Published online Feb. 12, 2015.

COPYRIGHT (c) 2015 by the Society of Nuclear Medicine and Molecular Imaging, Inc. its commercial introduction in 2011, this new technology has been steadily expanding its installed base. Currently, 4 models -2 fully integrated and 2 sequential-are commercially available for clinical applications.

One of the main challenges of hybrid PET/MR imaging is attenuation correction. Indeed, a critical step for quantitative PET is determining and compensating for the signal attenuation introduced by the patient. In PET/CT scanners, this attenuation correction can be achieved adapting the $\mathrm{x}$-ray attenuation information provided by the CT in PET/CT scanners. In the case of PET/MR scanners, the attenuation is estimated by segmenting MR data to identify different tissue classes (e.g., fat, soft tissue, lung) $(1,2)$. It follows that the accuracy of reconstructed PET data will be determined by how many tissue classes can be correctly identified.

Of particular relevance is the correct identification of bone tissue, due to its distinctively high attenuation value. However, in contrast to CT, MR is intrinsically much less suited for the depiction of cortical bone structures: the low proton density (PD) ( $\sim 20 \%$ of water) and heterogeneous structure lead to weak and short-lived signals (T2, 390 $\mu$ s at 3T) (3). Atlas methods have been proposed to overcome this obstacle (4-6), but they are not well suited for patients with off-norm anatomy, a common occurrence in PET indications such as oncology, for which postsurgical examinations are frequent.

Conventional sequences with echo times in the millisecond range are therefore too slow for direct bone signal detection. In contrast, ultrashort-echo-time (UTE) sequences with center-out $\mathrm{k}$-space acquisition enable sufficiently fast data acquisition to capture the bone signals $(7,8)$. Several studies have been published discussing the technical feasibility of UTE imaging for PET/MR attenuation correction. Generally, long T2 suppression methods (e.g., echo subtraction, saturation prepulses, or multiple sequences) are necessary to separate the bone signal from soft tissue (9). Arguably, the most widely accepted approach of bone segmentation for MR-based attenuation correction is based on the postprocessing of dual-echo UTE images (10-17).

A recent development has been the publication of a new bone identification technique, based on 3-dimensional radial zero-echo-time (ZTE) imaging (18). This sequence (similar to pointwise encoding time reduction with radial acquisition (19) and, to a certain extent, SWeep Imaging with Fourier Transformation (20)) has been proven to provide high-resolution isotropic images, suitable for bone segmentation $-1.4 \mathrm{~mm}^{3}$ in this study. Furthermore, bone segmentation is achieved without the need of preparation pulses or multiple echoes, making it a time-efficient acquisition. Rather than obtaining contrast from T2 relaxation time differences, this method explores the use of PD differences. 
The goal of the present study was to evaluate ZTE acquisitions for skull bone identification in PET/MR. For this purpose, a PET/CT/MR trimodality setup has been used to acquire clinical patient datasets, providing both the MR sequences under study and the gold-standard CT reference.

\section{MATERIALS AND METHODS}

\section{Data Acquisition}

Data were acquired using a trimodality setup consisting of a Discovery 750w 3T MR system (GE Healthcare) located in a room adjacent to a Discovery 690 time-of-flight PET/CT system (GE Healthcare). Patients were transported between the 2 systems using a dedicated transfer device, enabling a consistent patient placement between the PET/CT and MR imaging systems (GE Healthcare) (21).

Fifteen patients referred for a clinical oncology PET/CT examination were enrolled in this study. The average patient age was $59 \pm 17 \mathrm{y}$ (range, 32-88 y), the average weight was $75 \pm 9 \mathrm{~kg}$ (range, 66-100 $\mathrm{kg}$ ), and the average body mass index was $25 \pm 3 \mathrm{~kg} / \mathrm{m}^{2}$ (range, $20-33$ $\mathrm{kg} / \mathrm{m}^{2}$ ). Sixty percent of the patients were men and $40 \%$ women. Eleven of the patients displayed artificial alterations of the skull (craniotomies and craniectomies at various locations). The present study did not involve any extra radiation dose delivered to the patients, since the used CT was part of the clinical routine PET/CT examination. This study was approved by the institutional ethics committee, and written informed consent was obtained from all patients before the examination.

The voluntary MR examination was performed during the resting time after the radiotracer injection, so the total time at the site for the patient (PET/CT/MR, compared with standard PET/CT) was not altered, allowing approximately $30 \mathrm{~min}$ of MR scan time. The MR protocol included a ZTE acquisition, as well as further sequences used for the anatomic referencing of whole-body PET findings and not relevant for the current study. Thirteen patients were scanned with a 32-channel head coil (MR Instruments Inc.) and the remaining 2 with a head and neck coil (geometry embracing method head and neck unit; GE Healthcare).

The present study was based on the new bone imaging paradigm recently proposed by Wiesinger et al., based on proton density (PD)weighted ZTE $(22,23)$. The acquisition lasted $172 \mathrm{~s}$, covering a 26-cm transaxial and axial field of view with a resolution of $1.4 \times 1.4 \times 1.4 \mathrm{~mm}$. Four excitations were acquired, with a flip angle of $1^{\circ}$ and $62.5-\mathrm{kHz}$ bandwidth. The k-space was parsed using a 3-dimensional radial trajectory. The missing region at the center of k-space was filled with a second radial acquisition, with a reduced number of spokes and bandwidth, similar to the so-called water- and fat-suppressed proton projection MRI method (24). Before reconstruction, the 2 acquisitions were merged with a linear transition in the overlap region, to prevent the formation of artifacts. The raw data were reconstructed offline using standard 3-dimensional gridding $(25,26)$.

The PET/CT acquisition followed the standard protocol for a clinical oncology study. The helical CT scan acquired for PET attenuation correction $(120 \mathrm{kV} ; 15-80 \mathrm{~mA}$ with automatic dose modulation; rotation time, $0.5 \mathrm{~s}$; helical thickness, $3.75 \mathrm{~mm}$; pitch, $39.37 \mathrm{~mm} /$ rotation; matrix size, $512 \times 512,1.4 \times 1.4 \times 3.3 \mathrm{~mm}^{3}$ ) was used in this study as ground truth for the evaluation of MR-based bone imaging. The subsequent PET acquisition is not relevant for the present study.

\section{Postprocessing}

A detailed description of the segmentation procedure to generate bone and tissue masks from the reconstructed ZTE volumes can be found in Wiesinger et al. (22). The procedure essentially consists of a first intensity equalization step, followed by a set of histogram-based thresholding operations: first, a logarithmic rescaling of the normalized image intensities is applied to enhance the bone tissue. Then, a mask of the main body object is obtained to eliminate the background and coil elements. Within this body region, the bone and air regions are defined by thresholding operations based on a gaussian fitting of the main histogram peaks. Simple thresholding was also used to identify internal air cavities. The precise threshold values were defined based on the mean and full width at half maximum values of the noise and signal peaks. The formulas to generate the thresholds based on these were defined empirically $( \pm 2.25 \times$ full width at half maximum) but remained constant for all patients.

\section{Data Analysis}

The segmentation results were quantitatively evaluated using the Jaccard distance between CT and ZTE bone masks. This overlap distance is equal to 1 (or 100\%) for maximal dissimilarity and to 0 (or $0 \%$ ) for complete equality:

$$
\mathrm{J}_{\delta}\left(\mathrm{I}_{1}, \mathrm{I}_{2}\right)=\frac{\left|\mathrm{I}_{1} \cup \mathrm{I}_{2}\right|-\left|\mathrm{I}_{1} \cap \mathrm{I}_{2}\right|}{\left|\mathrm{I}_{1} \cup \mathrm{I}_{2}\right|} .
$$

The CT datasets were automatically registered to the segmented ZTE volumes using the rigid registration tool of the Integrated Registration plugin in an Advantage Workstation (GE Healthcare).

Notice that the results of the Jaccard distance are dependent on the threshold used to generate the CT bone mask. We determined this threshold by plotting, for each patient, the Jaccard distance for a wide range of possible threshold values. The optimal threshold for each patient was recorded, and the average threshold over all patients was used in the subsequent analysis.

This study of the quality of ZTE bone masks, compared with CT, was performed both considering the entire head image and considering only the cranium (to be precise, the axial span of the neurocranium). Studying both areas separately is useful for 2 reasons: first, it removes from the measure effects such as dental artifacts and jaw and vertebral (nonrigid) misalignment. Second, it provides a better idea of the different performance that can be expected when using ZTE for the attenuation correction of brain PET studies, as opposed to head and neck imaging.

In terms of clinical evaluation, each CT and ZTE image pair was inspected by a dual board-certified radiologist and nuclear medicine physician using the Advantage Workstation Volume Viewer software. Axial, sagittal, and coronal 2-dimensional views, as well as volume renderings, were used to determine the anatomic accuracy of ZTE bone maps in comparison to the CT gold standard. Selected areas of interest were assessed: the calvaria was subdivided into frontal, parietal, temporal, and occipital areas. The inner ear (bone/ fluid interfaces), the mastoid (bone/air/fluid interfaces), and the orbit (bone/muscle/fat interfaces) were assessed. Calcifications within the brain found consistently in adults
FIGURE 1. Axial (A and $B)$ and sagittal views $(C$ and $D)$ of reconstructed ZTE dataset. Logarithmic intensity rescaling $(B$ and $D)$ was applied to enhance bone tissue and facilitate its separation from background and internal air cavities.

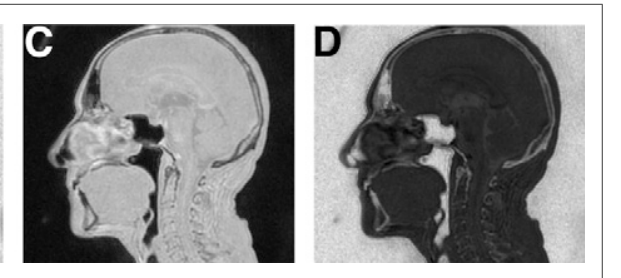




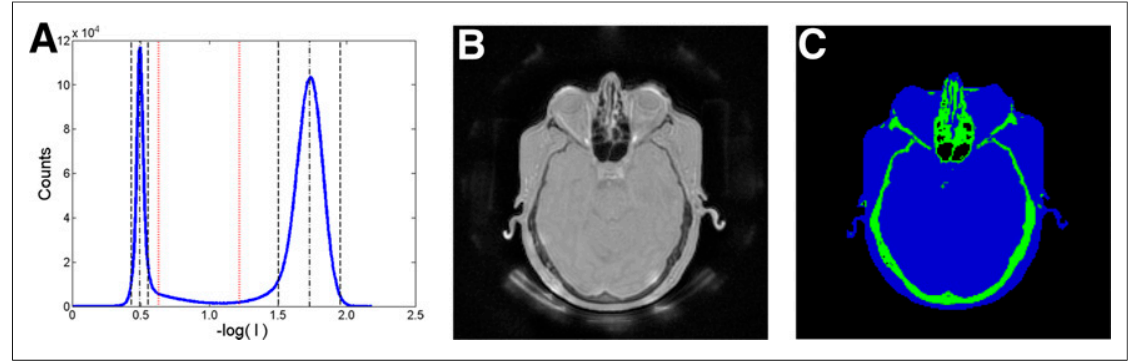

FIGURE 2. (A) Typical intensity histogram of ZTE dataset after logarithmic rescaling. Gaussian fitting result for soft-tissue and background peaks is indicated by dashed lines. Expected intensity range of bone tissue used in segmentation is indicated by dotted lines. (B) Axial view of corresponding ZTE volume. (C) Soft tissue (blue) and bone mask (green) obtained with segmentation.

low flip angles, an intrinsic property of ZTE imaging, has in this case the benefit of minimizing saturation effects in tissues with long relaxation times. In consequence, the low-intensity range of the image is occupied by little more than air and bone tissue, which is highlighted by the logarithmic intensity rescaling of the images (Figs. $1 \mathrm{~B}$ and 1D).

An example of the rescaled intensity histogram of a patient ZTE dataset can be found in Figure 2A. After intensity equalization, all datasets present clear peaks for the air and soft-tissue classes, easily identified by the automated gaussian fit-

were addressed as well (choroid plexus of lateral ventricles, pineal gland). Midline brain/fluid interfaces (third ventricle, fourth ventricle) were also part of the assessment. Another defined area of interest was artificial bone alterations, such as drilling holes or osteoplastic craniectomy sites.

\section{RESULTS}

Figures $1 \mathrm{~A}$ and $1 \mathrm{C}$ illustrate a typical single-echo ZTE dataset (in this case acquired with the geometry embracing method head and neck coil). Notice the flat contrast, uncommon in diagnostic imaging, intended to facilitate the segmentation. The use of very

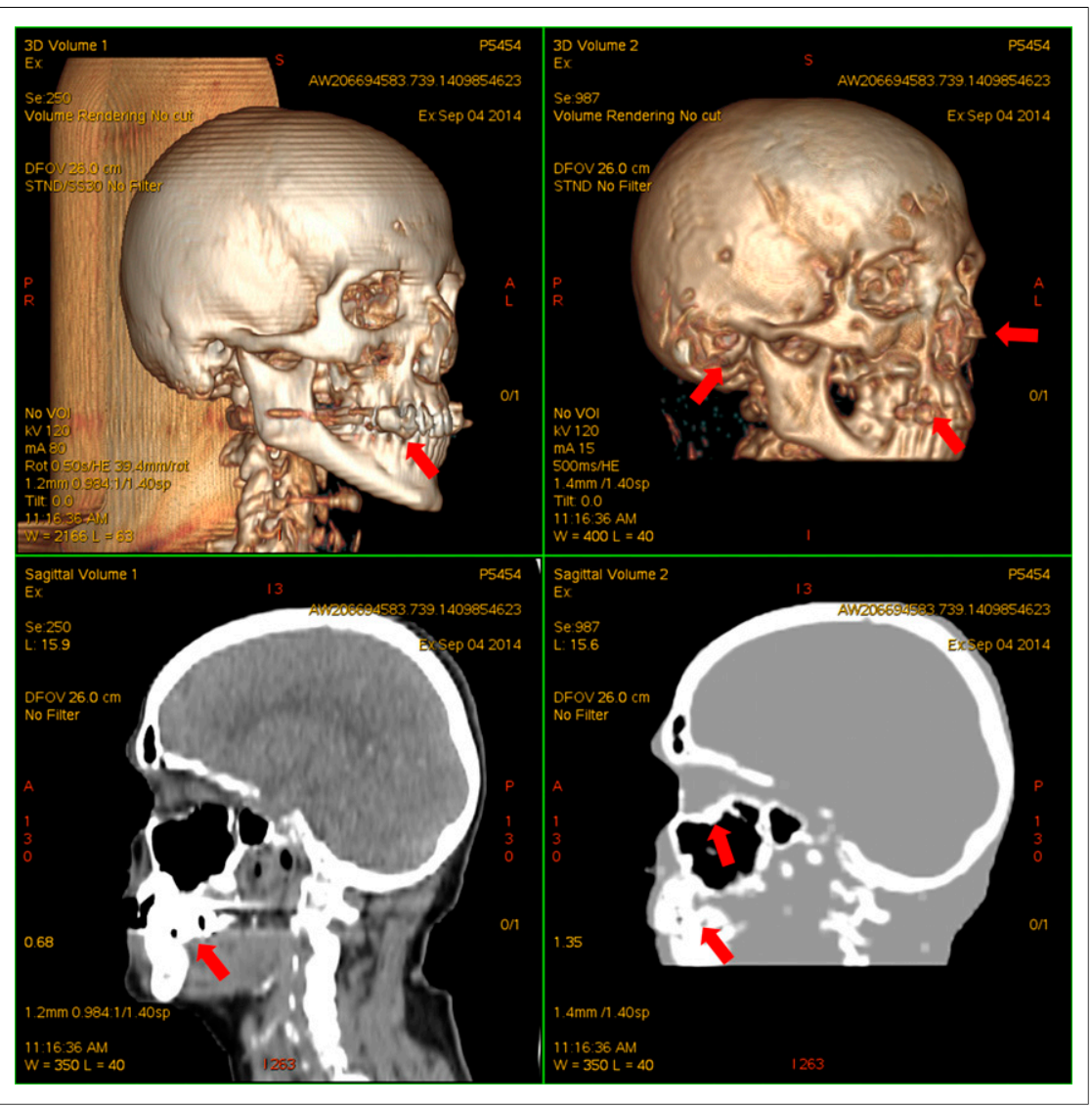

FIGURE 3. Volume rendering (top) and sagittal views (bottom) of CT dataset (left) and corresponding segmented ZTE dataset (right). Dental artifacts on both images, minor misclassification of cartilage and auditory canal air, and oversegmentation around sinusal cavities exist. ting. Figures $2 \mathrm{~B}$ and $2 \mathrm{C}$ show a typical segmentation result, obtained using the average and SD of these peaks to define the expected intensity range of bone tissue.

Figure 3 shows a side-by-side comparison of a segmented ZTE volume with the corresponding (registered) CT. Supplemental Figures 1 and 2 (supplemental materials are available at http://jnm. snmjournals.org) provide further examples, including artificial alterations of the skull.

Before the quantitative evaluation of the segmentation results, a suitable threshold had to be determined for the generation of bone maps from the reference CT datasets. Figure 4 shows the plot of Jaccard distances as a function of the selected CT threshold, for all patients. The optimal CT threshold was found to be $471 \pm 88$ Hounsfield units (HUs) (range, 356-636 HUs). As can be appreciated in the figure, it was a relatively wide and flat minimum in all cases. The difference in the Jaccard distance, when using this average threshold (471 HUs) rather than the individual optimum for each patient, was found to be no larger than $+1 \%$.

Table 1 shows the Jaccard distance measured between the CT and ZTE bone masks, for all patients. The measurements were performed twice, once considering the entire imaged area and again considering only the cranium. The average distance for the entire area was $52 \% \pm 6 \%$ (range, 38\%-63\%) and for the cranium only $39 \% \pm 4 \%$ (range, $32 \%-49 \%$ ). A visual representation of the agreement between CT and ZTE bone masks can be found in Figure 5.

As with UTE segmentation approaches, partial-volume effects on tissue/air interfaces led to false-positive bone voxels. These are accounted for by the segmentation procedure and eliminated using a Euclidean distance transform (similar to an erosion operation). However, some falsepositive voxels remain in isolated areas such as the auditory canal and nasal fossae. The fraction of false-positive voxels was measured to be $80 \% \pm 6 \%$. Most of these corresponded to high-density CT regions, 


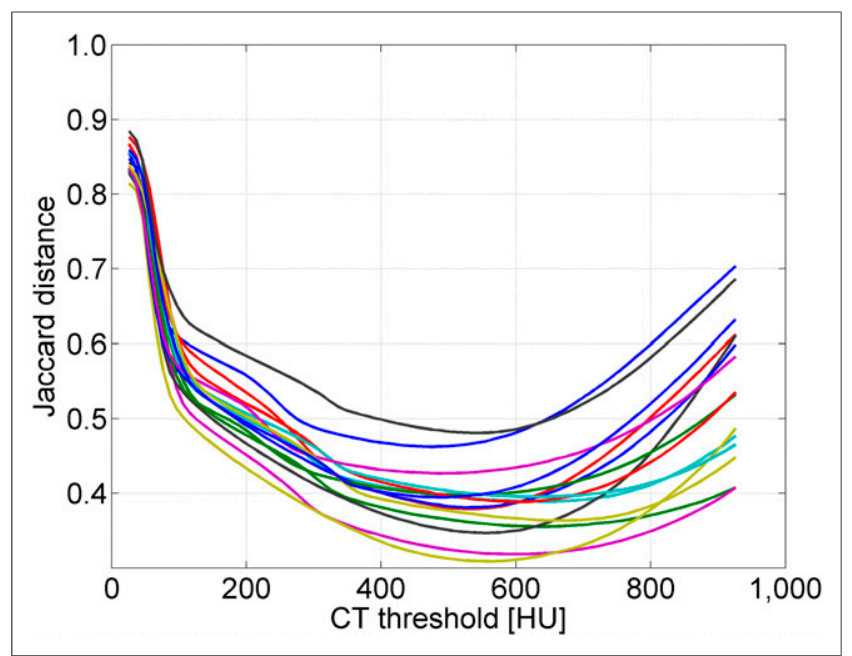

FIGURE 4. Jaccard distance between CT and ZTE bone masks, as function of $C T$ threshold value. Each line in plot corresponds to 1 of the patients.

close to but below the segmentation threshold. The percentage of bone voxels being classified as air and vice versa was in all cases below $1 \%$.

The results of the comparative clinical scoring of the ZTE bone maps are summarized in Table 2. Notice that these observations are specific to segmented bone maps, and some of the structures found missing there are still visible in the unsegmented ZTE data.

The calvaria yielded mainly minor issues, irrespective of the site. However, a few major issues were found in all calvaria sites, mainly frontally, consisting of apparent osteolysis on ZTE without correlative on CT. The apparent osteolysis was found to be caused by bone being misclassified as soft tissue. In most cases, this misclassification was limited to clusters of less than 5 voxels, often in regions with thin cortical bone layers separated by an air cavity or thick layer of trabecular bone. The misclassification seldom affected both layers of cortical bone (creating a perforation), and the overall morphology and continuity of the cranium were preserved in all cases. Bone union ridges and vessel foramina were visible in several cases.

Bone/fluid interfaces with high anatomic detail, as found in the inner ear, were consistently found to be a major issue for ZTE. Furthermore, localized misclassifications of bone as air were noticed in some regions in which bone tissue was too dense to yield sufficient MR signal. The mastoid air cells, although being complex anatomic structures as well, were found to pose fewer issues.

Almost no major issues were found in the orbit. Only in 1 subject, pseudoossifications were found in the conal space on ZTE. Localized false-positive bone voxels were occasionally found on the cornea.

The physiologic intracranial calcifications in the pineal gland (respectively, choroid plexus of the lateral ventricles) were depicted on ZTE in almost all subjects (respectively, half of the subjects). Voxels incorrectly labeled as hard material were displayed on ZTE images in the third ventricle more often than in the fourth ventricle. No cases were found in which brain structures were misclassified as air.

Contrary to previously reported skull segmentation studies based on UTE sequences (27), the presence of tendons on the bone masks obtained with ZTE was minor. Nasal cartilage was partially included in the bone masks.

In artificial osseous alterations (11/15 subjects), minor issues were found in 6 subjects and major issues in 3. Those major issues consisted of apparent osteolysis (bone to tissue misclassification, 2 subjects) and air (bone to air misclassification, 1 subject), for which reimplanted bone had a normal appearance on CT. Metal artifacts due to dental implants led to unwanted structures in the bone mask, corresponding to the edges of the MR signal void.

\section{DISCUSSION}

To our knowledge, this is the first clinical evaluation of skull bone identification based on a ZTE sequence, comparing the obtained bone masks with corresponding CT data. We have shown that single-echo PD-weighted acquisitions are well suited for automated bone identification. Furthermore, the quantitative and qualitative evaluation of the obtained bone masks compare positively with alternative methods found in the literature.

Our results are in agreement with those reported in the methodologic paper by Wiesinger et al. (22). They confirm that ZTE MR imaging is well suited for the depiction of tissues with fast decaying resonance signals and, in particular, for the efficient acquisition of PD-weighted datasets. These can be robustly segmented into air, soft-tissue, and bone compartments.

Relying on single-echo PD imaging is more time-efficient than the multiecho/multisequence T2* estimation approaches commonly found in the prior work (10-17). The saved time can be reinvested in the acquisition of higher resolution datasets, further improving the resulting bone maps. The ability to obtain images of higher resolution is restricted by the intrinsic signal-to-noise limitations of low flip-angle ZTE acquisition, leading to a tradeoff between the achievable resolution and the need for additional averaging.

The inverse logarithmic scaling of ZTE datasets provides a suitable means of direct inspection of the bone tissue information captured by the sequence. The image background and

TABLE 1

Jaccard Overlap Distances Measured Between ZTE and CT Bone Masks

\begin{tabular}{ccc}
\hline & \multicolumn{2}{c}{ Distance } \\
\cline { 2 - 3 } Patient & Whole & Cranium \\
\hline 1 & $59 \%$ & $46 \%$ \\
\hline 2 & $50 \%$ & $37 \%$ \\
\hline 3 & $53 \%$ & $38 \%$ \\
\hline 4 & $51 \%$ & $40 \%$ \\
\hline 5 & $46 \%$ & $33 \%$ \\
\hline 7 & $38 \%$ & $32 \%$ \\
\hline 8 & $57 \%$ & $36 \%$ \\
\hline 9 & $54 \%$ & $39 \%$ \\
\hline 10 & $63 \%$ & $40 \%$ \\
\hline 11 & $58 \%$ & $40 \%$ \\
\hline 12 & $46 \%$ & $41 \%$ \\
\hline 13 & $53 \%$ & $43 \%$ \\
\hline 14 & $50 \%$ & $38 \%$ \\
\hline 15 & $52 \%$ & $49 \%$ \\
\hline Mean & $46 \%$ & $40 \%$ \\
\hline Maximum & $52 \%$ & $39 \%$ \\
\hline Minimum & $63 \%$ & $49 \%$ \\
\hline SD & $38 \%$ & $32 \%$ \\
\hline
\end{tabular}




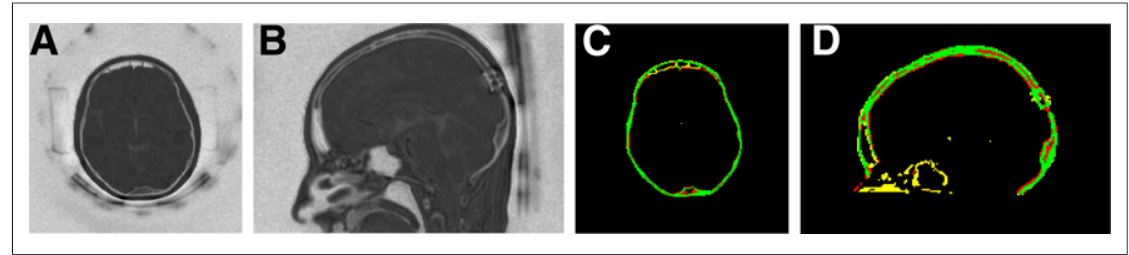

FIGURE 5. Logarithmic rescaling of ZTE dataset (A and B) and corresponding map of agreement between ZTE and CT bone masks ( $C$ and $D)$. Green pixels indicate true-positive bone identification, red pixels indicate false-negative (missed bone), and yellow pixels indicate false-positive. Residual misregistration can be perceived in, for example, occipital region. Notice surgical bone alteration.
Overall, the anatomic detail of ZTE bone masks was insufficient for direct clinical inspection but acceptable for attenuationcorrection purposes. Despite air cavity differentiation working remarkably well because of the PD contrast of the sequence, the problem of false-positive bone identification in certain interfaces affected by partial-volume effects remains.

The general morphology of the cranium was correctly captured in the ZTE masks. Apparent osteolyses were found, especially partial-volume effects in the air-tissue interfaces must be removed before rendering tools can be used.

The bone masks yielded by ZTE postprocessing were evaluated relative to CT, the current gold standard for bone imaging. Quantitative evaluation using the Jaccard distance shows better performance than previously reported for dual-echo UTE (27), which ranged between $47 \%$ and $79 \%$, as opposed to the $38 \%-63 \%$ obtained in this study.

The wide minima found during the optimization of the threshold for CT bone segmentation suggest that the bone structures detectable with ZTE are relatively well defined in terms of CT intensity (i.e., there are no undetected structures with similar attenuation properties, nor an excess of gradual intensity transitions). Hence, the ZTE bone mask is well suited for the purpose of attenuation modeling. Still, future work will aim at improving the processing of ZTE datasets to yield nonbinary estimations of tissue density. in the frontal calvaria. These were due to local (typically $<1 \mathrm{~cm}$ ) misclassification of thin trabecular bone layers as soft tissue. Often, only 1 layer was affected, suggesting that the misclassification would have a limited impact on attenuation estimation. Block effects related to the implementation of the intensity inhomogeneity correction were also identified as an occasional source of local bone misclassification. This problem will need to be addressed in future studies.

Structural detail in the inner ear was almost completely lost in ZTE bone masks of all patients, compared with CT (severely limiting their use for clinical diagnosis). Although structural detail was also diminished in the mastoid, with loss of some thin air cell septa, this did not affect the overall interpretation of the scans, because no relevant information was lost. More problematic, from the point of view of attenuation estimation, would be the misclassification of dense bone tissue as air. This is probably an inherent

TABLE 2

Clinical Scoring of ZTE Bone Masks in Comparison with CT Gold Standard

\begin{tabular}{|c|c|c|c|c|c|c|c|c|c|c|c|c|}
\hline \multirow[b]{3}{*}{ Patient } & & & & & \multirow{3}{*}{$\begin{array}{l}\text { Inner } \\
\text { ear }\end{array}$} & \multirow[b]{3}{*}{ Mastoid } & \multirow[b]{3}{*}{ Orbit } & \multicolumn{2}{|c|}{$\begin{array}{l}\text { Cerebrospinal } \\
\text { fluid space }\end{array}$} & \multirow{3}{*}{$\begin{array}{l}\text { Choroid } \\
\text { plexus } \\
\text { of lateral } \\
\text { ventricle } \\
\text { (calcification) }\end{array}$} & \multirow{3}{*}{$\begin{array}{l}\text { Pineal gland } \\
\text { (calcification) }\end{array}$} & \multirow{3}{*}{$\begin{array}{l}\text { Artificial } \\
\text { alteration }\end{array}$} \\
\hline & \multicolumn{4}{|c|}{ Calvaria } & & & & Third & Fourth & & & \\
\hline & Frontal & Parietal & Temporal & Occipital & & & & ventricle & ventricle & & & \\
\hline 1 & 1 & 0 & 1 & 1 & 2 & 1 & 1 & 1 & 1 & 1 & 0 & NA \\
\hline 2 & 1 & 1 & 1 & 0 & 2 & 1 & 0 & 0 & 0 & 1 & 0 & 1 \\
\hline 3 & 0 & 1 & 1 & 1 & 2 & 1 & 1 & 2 & 0 & 1 & 1 & 1 \\
\hline 4 & 0 & 1 & 1 & 1 & 1 & 1 & 0 & 2 & 0 & 1 & 0 & 1 \\
\hline 5 & 1 & 1 & 0 & 1 & 1 & 1 & 2 & 2 & 0 & 1 & 0 & 0 \\
\hline 6 & 1 & 1 & 1 & 1 & 1 & 1 & 1 & 1 & 0 & 0 & 0 & 2 \\
\hline 7 & 2 & 0 & 1 & 1 & 2 & 1 & 1 & 2 & 1 & 0 & 0 & 0 \\
\hline 8 & 2 & 1 & 1 & 1 & 2 & 1 & 0 & 0 & 0 & 0 & 1 & 1 \\
\hline 9 & 1 & 1 & 1 & 1 & 2 & 1 & 1 & 0 & 1 & 0 & 0 & NA \\
\hline 10 & 1 & 1 & 1 & 1 & 2 & 1 & 1 & 2 & 0 & 1 & 0 & NA \\
\hline 11 & 1 & 2 & 2 & 2 & 2 & 1 & 1 & 0 & 0 & 1 & 0 & 2 \\
\hline 12 & 1 & 1 & 1 & 1 & 1 & 1 & 0 & 0 & 0 & 0 & 0 & NA \\
\hline 13 & 1 & 0 & 1 & 1 & 2 & 1 & 0 & 2 & 0 & 1 & 0 & 1 \\
\hline 14 & 2 & 2 & 2 & 1 & 2 & 1 & 1 & 2 & 0 & 1 & 0 & 2 \\
\hline 15 & 1 & 0 & 1 & 1 & 2 & 1 & 0 & 2 & 2 & 1 & 0 & 1 \\
\hline Mean & 1.1 & 0.9 & 1.1 & 1.0 & 1.7 & 1.0 & 0.7 & 1.2 & 0.3 & 0.7 & 0.1 & 1.1 \\
\hline SD & 0.6 & 0.6 & 0.5 & 0.4 & 0.5 & 0.0 & 0.6 & 0.9 & 0.6 & 0.5 & 0.4 & 0.7 \\
\hline
\end{tabular}

$1=$ minor issue; $0=$ no issue; 2 = major issue; $\mathrm{NA}=$ not applicable. 
limitation of the acquisition, due to the low density of signal-emitting tissue. Regardless of the highly localized nature of this issue, further study will be required to assess its impact in attenuation correction.

Fat/muscle/bone interfaces such as in the retrobulbar region yielded satisfactory results, except in 1 subject in whom apparent conal ossification on ZTE was possibly inferred by eyeball movements.

We could show that even small calcifications, such as in the pineal gland, are reliably interpreted as hard structures by ZTE. The incorrect hard structures displayed by ZTE in the third ventricle were likely induced by a combination of magnetization saturation and the thin, slitlike configuration of this cerebrospinal fluid space, with 2 brain/fluid interfaces being close, as opposed to the fourth ventricle, for which pseudocalcifications were found less commonly. Classification issues in the ventricles have also been reported for UTE-based methods (28). Anatomy-based postprocessing may need to be included in the segmentation to mitigate this issue.

Similarly, the throat and vertebrae area would benefit from dedicated processing, which could account both for the lower density bone tissue and for the false-positives in the oropharyngeal cavity interfaces.

Apparent lytic or even air-containing lesions in reimplanted bone on ZTE, being observed in 3 of 11 subjects with postoperative changes, do have grave clinical influence. Extreme caution should therefore be taken when interpreting ZTE datasets around such areas. Signal voids around implanted metallic objects are a serious limitation of the proposed method, and correction approaches are currently being investigated.

Further work will be directed at extending the use of ZTE bone segmentation to whole-body applications. Other potential applications of the ZTE sequence for PET/MR imaging would be lung visualization, to improve the estimation of parenchyma attenuation (29) and the detection of local coils.

\section{CONCLUSION}

This is the first, to our knowledge, clinical evaluation of skull bone identification based on a ZTE sequence. The results suggest that single-echo, PD-weighted ZTE imaging is an efficient means of obtaining anatomically accurate maps of bone tissue for the purpose of PET attenuation correction. The segmentation results show better bone depiction and separation from air cavities and collagenous tissue than previously reported methods. Further work will be aimed at obtaining nonbinary estimations of bone density.

\section{DISCLOSURE}

The costs of publication of this article were defrayed in part by the payment of page charges. Therefore, and solely to indicate this fact, this article is hereby marked "advertisement" in accordance with 18 USC section 1734 . No potential conflict of interest relevant to this article was reported.

\section{REFERENCES}

1. Martinez-Möller A, Souvatzoglou M, Delso G, et al. Tissue classification as a potential approach for attenuation correction in whole-body PET/MRI: evaluation with PET/CT data. J Nucl Med. 2009;50:520-526.

2. Hofmann M, Pichler B, Scholkopf B, Beyer T. Towards quantitative PET/MRI: a review of MR-based attenuation correction techniques. Eur J Nucl Med Mol Imaging. 2009;36(suppl 1):S93-S104.

3. Du J, Carl M, Bydder M, Takahashi A, Chung CB, Bydder GM. Qualitative and quantitative ultrashort echo time (UTE) imaging of cortical bone. J Magn Reson. 2010;207:304-311
4. Hofmann M, Steinke F, Scheel V, et al. MRI-based attenuation correction for PET/MRI: a novel approach combining pattern recognition and atlas registration. J Nucl Med. 2008;49:1875-1883.

5. Qian H, Shanbhag D, Kaushik S, et al. Whole-body PET/MR attenuation correction combining image segmentation, truncation completion and atlas-based skull segmentation. Paper presented at: PET/MR and SPECT/MR: New Paradigms for Combined Modalities in Molecular Imaging Conference; May 26-30, 2012; Elba, Italy.

6. Malone IB, Ansorge RE, Williams GB, Nestor PJ, Carpenter TA, Fryer TD. Attenuation correction methods suitable for brain imaging with a PET/MRI scanner: a comparison of tissue atlas and template attenuation map approaches. J Nucl Med. 2011;52:1142-1149.

7. Robson MD, Bydder GM. Clinical ultrashort echo time imaging of bone and other connective tissues. NMR Biomed. 2006;19:765-780.

8. Robson MD, Gatehouse PD, Bydder M, Bydder GM. Magnetic resonance: an introduction to ultrashort TE (UTE) imaging. J Comput Assist Tomogr. 2003;27:825-846.

9. Du J, Bydder M, Takahashi AM, Carl M, Chung CB, Bydder GM. Short T2 contrast with three-dimensional ultrashort echo time imaging. Magn Reson Imaging. 2011;29:470-482.

10. Keereman V, Fierens Y, Broux T, De Deene Y, Lonneux M, Vandenberghe S. MRI-based attenuation correction for PET/MRI using ultrashort echo time sequences. J Nucl Med. 2010;51:812-818.

11. Wang L, Zhong X, Zang L, Tiwari D, Mao H. Ultra-short TE (UTE) imaging of skull and a quantitative comparison of skull images obtained from MRI and CT. Proc. Intl. Soc. Mag. Reson. Med. 2010;18:796.

12. Catana C, Van der Kouwe A, Benner T, et al. MR-Based PET attenuation correction for neurological studies using dual-echo UTE sequences. Proc. Intl. Soc. Mag. Reson. Med. 2010;18:3953.

13. Catana C, van der Kouwe A, Benner T, et al. Toward implementing an MRIbased PET attenuation-correction method for neurologic studies on the MR-PET brain prototype. J Nucl Med. 2010;51:1431-1438.

14. Johansson A, Karlsson M, Nyholm T. CT substitute derived from MRI sequences with ultrashort echo time. Med Phys. 2011;38:2708-2714.

15. Berker Y, Franke J, Salomon A, et al. MRI-based attenuation correction for hybrid PET/MRI systems: a 4-class tissue segmentation technique using a combined ultrashort-echo-time/Dixon MRI sequence. J Nucl Med. 2012;53:796-804.

16. Navalpakkam BK, Braun H, Kuwert T, Quick HH. Magnetic resonance-based attenuation correction for PET/MR hybrid imaging using continuous valued attenuation maps. Invest Radiol. 2013;48:323-332.

17. Delso G, Zeimpekis K, Carl M, Wiesinger F, Hüllner M, Veit-Haibach P. Clusterbased segmentation of dual-echo ultra-short echo time images for PET/MR bone localization. EJNMMI Physics. 2014;1:1-13.

18. Madio DP, Lowe IJ. Ultra-fast imaging using low flip angles and FIDs. Magn Reson Med. 1995;34:525-529.

19. Grodzki DM, Jakob PM, Heismann B. Ultrashort echo time imaging using pointwise encoding time reduction with radial acquisition (PETRA). Magn Reson Med. 2012;67:510-518.

20. Idiyatullin D, Corum C, Park JY, Garwood M. Fast and quiet MRI using a swept radiofrequency. J Magn Reson. 2006;181:342-349.

21. Veit-Haibach P, Kuhn FP, Wiesinger F, Delso G, von Schulthess G. PET-MR imaging using a tri-modality PET/CT-MR system with a dedicated shuttle in clinical routine. MAGMA. 2013;26:25-35.

22. Wiesinger F, Sacolick L, Kaushik S, Ahn S, Delso G, Shanbhag D. Zero TE bone imaging. Paper presented at: ISMRM; May 10-16, 2014; Milan, Italy.

23. Wiesinger F, Sacolick LI, Menini A, et al. Zero TE MR bone imaging in the head. Magn Reson Med. January 16, 2015 [Epub ahead of print].

24. Wu Y, Ackerman JL, Chesler DA, Graham L, Wang Y, Glimcher MJ. Density of organic matrix of native mineralized bone measured by water- and fat-suppressed proton projection MRI. Magn Reson Med. 2003;50:59-68.

25. Jackson JI, Meyer CH, Nishimura DG, Macovski A. Selection of a convolution function for Fourier inversion using gridding. IEEE Trans Med Imaging. 1991;10:473-478.

26. Beatty PJ, Nishimura DG, Pauly JM. Rapid gridding reconstruction with a minimal oversampling ratio. IEEE Trans Med Imaging. 2005;24:799-808.

27. Delso G, Carl M, Wiesinger F, et al. Anatomic evaluation of 3-dimensional ultrashort-echo-time bone maps for PET/MR attenuation correction. $\mathrm{J} \mathrm{Nucl}$ Med. 2014;55:780-785.

28. Choi H, Cheon GJ, Kim H-J, et al. Segmentation-based MR attenuation correction including bones also affects quantitation in brain studies: an initial result of ${ }^{18} \mathrm{~F}-\mathrm{FP}-$ CIT PET/MR for patients with parkinsonism. J Nucl Med. 2014;55:1617-1622.

29. Gibiino F, Sacolick L, Menini A, Landini L, Wiesinger F. Free-breathing, zeroTE MR lung imaging. MAGMA. September 9, 2014 [Epub ahead of print]. 